\title{
Genetic Bases of Hypertrophic Cardiomyopathy
}

\author{
Beatriz Piva e Mattos
}

Porto Alegre, RS - Brazil

Hypertrophic cardiomyopathy is characterized by myofibrillar derangement and predominant hypertrophy of the interventricular septum associated with a reduced or normal left ventricular cavity ${ }^{1}$. It determines diastolic dysfunction and a tendency towards myocardial ischemia, arrhythmia, and sudden death. With a prevalence of 1 case per 500 individuals ${ }^{2}$, it occurs in all age groups, from birth to the $8^{\text {th }}$ decade, having a genetic origin preliminarily detected from familiar forms among carriers of the disease ${ }^{3}$.

The complex character of this entity outlined in the first reports of the modern era ${ }^{4.5}$ became unequivocally evident by systematic studies developed over recent decades ${ }^{3,6-9}$. The application of a methodology generated in molecular genetics by obtaining genetic maps of high resolution facilitated the redefinition of concepts related to the pathology, physiopathology, and diagnosis of hypertrophic cardiomyopathy. Genetic analysis facilitating identification of the disease in its preclinical phase and risk stratification based on molecular criteria render the future introduction of genetic therapy viable.

Hypertrophic cardiomyopathy has highly heterogeneous morphological, functional, and clinical characteristics. The phenotype is composed of ventricular hypertrophy associated with myofibril disorganization. Marked phenotype variation relative to the magnitude and extension of the myocardial hypertrophy is observed. Echocardiographic evaluation reveals predominant hypertrophy of the intraventricular septum of diffuse character, commonly extending to the free anterior-lateral wall of the left ventricle ${ }^{8,9}$. Wall thickness is usually above $20 \mathrm{~mm}$, although measurements between 20 and $15 \mathrm{~mm}$ have been found ${ }^{9}$. Borderline thicknesses of $15 \mathrm{~mm}$ or even $13 \mathrm{~mm}$ have also been identified ${ }^{10-12}$. Asymmetric forms predominate over concentric ones, representing 1 to $2 \%$ of the cases $^{7}$. Myofibrillar disarray extends over 5 to $30 \%$ of the

Hospital de Clínicas de Porto Alegre - Faculdade de Medicina da UFRGS Mailing Address: Beatriz Piva e Mattos - Rua Ramiro Barcelos, 2350 - 90035-003 Porto Alegre, RS, Brazil - E-mail: hepa@hepa.ufrgs.br myocardial tissue and has a low correlation with the degree of hypertrophy ${ }^{11}$.

Hypertrophic cardiomyopathy can appear without showing its main morphological characteristics. Arrhythmias and sudden death in the absence of echocardiographic signals of left ventricular hypertrophy $(\mathrm{LVH})$ in patients with the genotype, indicate incomplete phenotype expression ${ }^{13}$. It is concluded that the phenotype is represented by a continuous spectrum, from macroscopically normal to severe forms showing massive myocardial hypertrophy ${ }^{14-16}$.

The phenotype changes with age. Hypertrophic myopathy can appear at birth showing serious obstructive forms with marked hypertrophy, rapid evolution to cardiac failure, and a high level of mortality ${ }^{17}$. It more frequently manifests clinically in adolescence when a detectable increase in parietal thickness of the left ventricle accompanies an increase in weight and stature ${ }^{11}$. Although clinical evolution in children and adolescents is usually benign, annual mortality can reach $4.8 \%$, higher than that shown among adults ${ }^{18}$. Adolescents and young adults can manifest extreme degrees of LVH with outflow tract obstruction, cavity reduction, and evolution towards sudden death ${ }^{11,18}$. An inverse relationship between age group and left ventricular parietal thickness may be shown ${ }^{11}$. Gradual regression in hypertrophy, increased ventricle volume, and diastolic dysfunction occur in approximately $10 \%$ of individuals reaching maturity ${ }^{19}$. Late forms, manifest after 65 years of age, are individualized, with a hypertrophy generally restricted to the interventricular septum ${ }^{20}$.

The natural history is also heterogeneous. While some cases remain in asymptomatic form indefinitely, other patients experience sudden death or evolve to cardiac failure. Although annual mortality recorded at reference centers falls between 2 and $6 \%{ }^{18,21}$, measurements among less selected populations fall between 0.5 and $1.5 \%$ of ca$\operatorname{ses}^{22-24}$. Clinical indicators like degree of hypertrophy, records of ventricular arrhythmia, and abnormal response to exercise, have a limited value for risk stratification of sudden death ${ }^{25,26}$. 
The clinical complexity characterizing hypertrophic cardiomyopathy becomes even more evident when its genetic aspects are analyzed. Transmission by autosomal dominant heritage attributes the same genetic disturbance to all affected members of a family ${ }^{21}$. Although preliminary verification has estimated that only $50 \%$ of the cases show a familial character ${ }^{27}$, more recent evaluations consider that truly sporadic forms are borne by less than $10 \%$ of disease carriers ${ }^{14,28}$. Three factors may underestimate the diagnosis of genetic forms: evaluation of very small families, adoption of rigid echocardiographic criteria, and the presence of mutations of variable penetrance ${ }^{10,15,29}$. Echocardiograhic manifestations of LVH may be absent or have a subclinical character, especially among children and adolescents. The electrocardiogram may be useful for identifying these patients. About $50 \%$ of adult carriers of the genotype who have normal wall thickness on the echocardiogram have electrocardiographic alterations ${ }^{10}$.

The large clinical variability among families affected by hypertrophic cardiomyopathy suggests the involvement of more than 1 gene with phenotypic manifestations in common. In 1989, Jarcho et $\mathrm{al}^{30}$, using genetic linkage analysis, mapped on chromosome 14q1, the first locus related to the disease, later described as CMH 1. One year later, GeisterferLowrance et $\mathrm{ll}^{31} \mathrm{using}$ genetic mapping and DNA sequencing individualized the point mutation affecting the gene that codifies the $\beta$-miosin heavy chain, considered to cause the disease.

Up to the present time, 7 further genes have been related to hypertrophic cardiomyopathy; they encode, respectively, cardiac troponin $\mathrm{C}^{32}, \alpha$ tropomyosin ${ }^{33}$, myosinbinding protein $C^{34,35}$, essential and regulatory myosin light chains ${ }^{36}$, cardiac tropomyosin $\mathrm{I}^{37}$, and cardiac $\alpha$ actin ${ }^{38}$ (Table I). Reports of a mutation involving the gene of titin and of an as yet unidentified gene at locus 7q3 in forms associated with the Wolff-Parkinson-White syndrome ${ }^{39}$ are still under evaluation. The genetic heterogeneity would partially justify the clinical variability among carriers of the disease. The finding that phenotype expression has intrafamilial variability suggests the participation of other factors, genetic or environmental.

Although from the biomolecular viewpoint, hyper-

\begin{tabular}{|c|c|}
\hline \multicolumn{2}{|c|}{$\begin{array}{c}\text { Table I - Genes related to hypertrophic cardiomyopathy and } \\
\text { chromosome location }\end{array}$} \\
\hline Gene & Chromosome \\
\hline Cardiac $\beta$ myosin heavy chain & $14 \mathrm{q} 1$ \\
\hline Cardiac $\mathrm{T}$ troponin & $1 \mathrm{q} 32$ \\
\hline$\alpha$ tropomyosin & $15 \mathrm{q} 22$ \\
\hline Myosin binding $\mathrm{C}$ protein & $11 \mathrm{p} 11.2$ \\
\hline Essential myosin light chain & $3 \mathrm{p} 21.2-\mathrm{p} 21.3$ \\
\hline Regulatory myosin light chain & $12 \mathrm{q} 23-\mathrm{q} 24.3$ \\
\hline Cardiac I troponin & $19 \mathrm{p} 13.2-\mathrm{q} 13.2$ \\
\hline$\alpha$ cardiac actin & 15 \\
\hline
\end{tabular}

trophic cardiomyopathy can be interpreted as a multiple entity, the fact that the genes affected encode sarcomere proteins gives a unifying character to the entity ${ }^{16}$. About 100 mutations have been described, corresponding to $2 / 3$ of the total ${ }^{16}$. One, therefore, observes allelic heterogeneity, several mutations involving the same gene being capable of evoking the disease.

In the human genome, approximately 30,000 genes encode structural proteins and enzymes. They constitute DNA segments formed by nucleotide sequences with a particular location in the chromosome, called the chromosome locus. A nitrogen base, the deoxyribose sugar, and a phosphate group form the nucleotides. Two types of nitrogen bases exist, purines [adenine (A) and guanine $(\mathrm{G})]$ and pyrimidines [cytosine (C) and thymine (T)]. Genes have a variable dimension, from a few hundred to thousands of base pairs. They have paternal and maternal copies called alleles, and their production results from the combination of both. The base-pair sequence in DNA is used to form messenger RNA (mRNA), which takes information to the ribosomes localized in the cytoplasm where protein synthesis takes place. The RNA molecule is synthesized directly from a DNA template, a process called transcription. Human genes have base sequences called exons that encode amino acids and are transcribed into mRNA and nonfunctional sequences, the introns. At the final step of the formation of mRNA, the intron sequences are removed and the exons united by a process known as splicing, taking place in ribonucleic corpuscles. The removal of the intron sequences is performed using the donor and acceptor sites located at the exon-intron borders. The base sequence of the mRNA will determine the amino acid sequence of the proteins. If an error occurs during the splicing process, a modified protein is formed. The genetic code contained in the DNA helix and in the mRNA is essential for the formation of the different types of proteins. Base triplets, the codons, form it. Sixty-four codons exist, of which 61 encode amino acids and 3 are termination codons, which have no encoding function. The genetic code describes the relationship between the base sequences and the amino acid sequence of the protein. Point mutations determine permanent changes in the base sequence of a DNA molecule by substituting a pair of bases with a reflex on the encoded protein ${ }^{40}$.

\section{Mutations of the gene of the cardiac $\beta$-miosin heavy} chain - About $35 \%$ of the cases of hypertrophic cardiomyopathy are caused by mutations of the gene of the heavy chain of cardiac b myosin ${ }^{16,41}$, which encodes the predominant isoform expressed in the ventricles. The thick filaments contain 2 proteins of the cardiac $\beta$ myosin heavy chain that are connected to 4 molecules of the light myosin chains. The first contains $30 \%$ cardiac myosin, comprising $95 \%$ ventricle myosin ${ }^{28}$. It is a contractile protein with enzymatic 
action, which in the presence of actin hydrolyzes ATP into ADP and phosphate. The molecule formed by 1,934 amino acids has an asymmetric shape and distinct segments. The globular head contains the actin binding sites and ATP. The neck region is associated with to the light myosin chains, and the rod like tail, in turn, establishes the connection to myosin molecules that form the thick filaments, each made up of 400 molecules ${ }^{28,42}$.

In their near-totality, mutations that affect the gene of the cardiac $\beta$ myosin heavy chain are point mutations of the type described as "missense mutations" in which the substitution of a single base of the DNA in a given exon results in the exchange of the encoded amino acid ${ }^{15}$. Deletion of the nontranslated 3' region of the gene, inducing the loss of at least 5 amino acids in the rod of the protein has also been reported ${ }^{43}$. Deletions in codons 10 and 930, which do not result in a change in the DNA reading frane, have been subsequently described ${ }^{42}$.

At least 50 mutations of the gene of the cardiac $\beta$ myosin heavy chain have been identified affecting unrelated families. It is estimated that the greater part has been already individualized. All cause common manifestations of hypertrophic cardiomyopathy. The mutations described cause the disease and do not constitute polymorphism connected to adjacent genes, unrelated to the disease.

Mutations with changes in the encoded amino acid determine alterations in the protein structure of the gene product. The substitution of the amino acid takes place in the protein molecule. Mutated residues are localized at the interface with actin and the binding with ATP and the myosin light chains ${ }^{15}$. The mutant allele produces an anomalous protein that, when incorporated into the thick filaments, determines contractile dysfunction, even though the normal allele produces an integral protein. It is, therefore, a dominant negative mutation ${ }^{44}$ and may or may not determine the change in the charge of encoded amino acid, altering the biomolecular conformation of the polypeptide ${ }^{28}$.

The cardiac $\beta$ myosin heavy chain polypeptides are encoded in $30 \mathrm{~KB}$ of DNA, arranged in 40 exons; this renders the analysis of each one extremely laborious and time-consuming ${ }^{42}$. The near-totality of the missense mutations is localized in the first 23 exons, which encode the globular head and the head-tail junction of the protein ${ }^{45}$. This gene is highly susceptible to mutagenesis in special codons 403,719 , and $741^{46,47}$. It is suspected that the arginine residue plays a fundamental role in the protein's normal function ${ }^{46}$. However, no mutation has a predominant character, although Arg403Gln is a frequent one. The greater part of the mutations result from the exchange of guanine or cytosine ${ }^{28}$.

The mutations of the cardiac $\beta$ myosin heavy chain were the first to be described, allowing greater knowledge of the genotype-phenotype relationship, degree of penetrance, and clinical evolution. Yet, the analysis of these as- pects is based on the evaluation of a still limited number of cases, not rarely originating from a single family. The clinical valorization of the information presently available depends on confirmation at a higher scale, using data resulting from the identification of new affected families.

Mutations of the cardiac $\beta$ myosin heavy chain are frequently associated with expressive myocardial hypertrophy with high penetrance ${ }^{28}$. Although hypertrophy has a similar degree and extension in the diverse mutations of the gene, the prognosis shows variable characteristics. Consequently, risk stratification can only be determined on the basis of genetic analysis, because many mutations of the same gene have the phenotype in common.

Certain mutations in the cardiac $\beta$ myosin heavy chain are considered malignant due to the high incidence of sudden death. They are associated with a change in the charge of the encoded amino acid ${ }^{45}$. Benign mutation can also exhibit this characteristic. Malignant forms encode severe myocardial hypertrophy and complete penetrance. Arg403Gln, the first described mutation ${ }^{31}$ results from the exchange of adenine by guanine at exon 13 , resulting in the substitution of arginine by glutamine in codon 403. It causes premature sudden death, which in $50 \%$ of cases occurs before the age of $30^{45}$. The same mutation was described in a small Korean family with a low incidence of sudden death, despite its high penetrance ${ }^{48}$. Arg719GIn and Arg453Cys are equally serious, with a high incidence of sudden death and an average survival time of 38 and 30 years, respectively ${ }^{45,49}$. Gly716Arg, with a penetrance of $100 \%$, precocious phenotype expression, and premature sudden death, evolves towards cardiac failure in the adult (50).

Mutations Glu930Lys and Arg249Gln are of intermediate risk ${ }^{28,45}$. Leu908Val has a better prognosis: $92 \%$ of patients are alive at 60 years ${ }^{48}$. Gly256Glu like Arg 403Trp and Arg870His are also benign ${ }^{46,51,52}$. Mutation Val606Met is described as benign but can eventually have a bad prognosis ${ }^{45,52}$. Mutations involving the same codon, like Arg403Gln, Arg403Leu, and Arg 403Trp can have a distinct prognosis and a variable degree of penetrance ${ }^{15}$.

The analysis of many families with an identical mutation indicates that the mutations can have an independent origin. Mutations of the gene of the cardiac $\beta$ myosin heavy chain gene are also identified in carriers of sporadic forms ${ }^{53}$, demonstrating that familiar and nonfamiliar forms make up the same spectrum. It consists of spontaneous mutations transmitted to the descendants with expression on the mRNA and the carrier protein; this may not be so in the case of a very small family.

In mutations of the gene of the cardiac $\beta$ myosin heavy chain, because penetrance is usually high, it is an exception for carriers of the genotype not to exteriorize the phenotype until adolescence. The high penetrance conditions the carrier for severe hypertrophy ${ }^{15}$. Only a few muta- 
tions, like Arg403Leu, Arg403Trp and Asn232Ser have low penetration ${ }^{44,52}$. In these cases, hypertrophy may be mild or borderline ${ }^{44}$. Variation in the degree of hypertrophy is observed between families carrying the same mutation ${ }^{15}$.

The greater part of the mutated protein is expressed in the sarcomere of the myocardial fibers, directly affecting the generation of contractile force ${ }^{42}$. Analysis of endomyocardial biopsies reveals the loss of sarcomere filament alignment, conditioning to miofibrillar disarray ${ }^{54}$. The mutated myosin is also expressed in the skeletal musculature producing myopathy with a predominance of slow-type myofibrils and absence of mitochondria ${ }^{55}$.

Mutations of the cardiac troponin T gene -In 1993, Watkins et al ${ }^{32}$ individualized a second locus related to cardiac hypertrophy, situated in chromosome 1q32, and then named it CMH 2. One year after, the same workers described 3 mutations affecting the gene Troponin $\mathrm{T}$, mapped on this locus ${ }^{56}$.

Cardiac T troponin mutations represent about $15 \%$ of hypertrophic cardiomyopathy cases ${ }^{41}$. Various isoforms originated by recomposition are expressed in the myocardium. Thin filaments are formed by actin, tropomyosin and troponins $\mathrm{T}, \mathrm{I}$, and $\mathrm{C}^{42}$. Contraction depends on actinmyosin interaction, regulated by a tropomyosin and the troponin complex. Once calcium-C troponin binding is established, the troponin-tropomyosin complex releases actin, enabling the binding of the globular heads of cardiac $\beta$ myosin to the thin filaments.

The gene of cardiac $\mathrm{T}$ troponin is composed of 17 exons distributed over $17 \mathrm{~KB}$ of $\mathrm{DNA}^{42}$. Missense mutation in a total of 10 , localized in exons $8,9,11,14$, and 16 predominate $^{41,57}$. De novo mutation in exon 9, Arg 92 Trp, has recently been reported ${ }^{58}$. Deletion of 3 nucleotides corresponding to all of the codon of glutamic acid, $\Delta$ Glu160, was also identified ${ }^{41}$. Splice site mutations, involving the donor site of intron 15 , Int $15^{\mathrm{G}-\mathrm{A}}$, generates a truncated, rapidly degraded protein, the consequence of the loss of 28 terminal amino acids ${ }^{41}$. Preparations containing intact sarcomeres with a high expression of truncated cardiac troponin $\mathrm{T}$ show a marked reduction in their contractile function ${ }^{59}$. In the presence of low calcium concentrations, relaxation is impaired by interference with the inhibitor function of cardiac I troponin.

Clinical manifestations are less heterogeneous than those evidenced by mutations of the cardiac $\beta$ myosin heavy chain. For the most part, they determine similar phenotypes and show low penetrance. Hypertrophy generally of a mild or subclinical grade may be absent in $25 \%$ of the cases, according to echocardiographic findings ${ }^{41}$. Maximal wall thickness is between 11 and $16 \mathrm{~mm}$, in contrast to that produced by mutations in the cardiac $\beta$ myosin heavy chain, average $24 \mathrm{~mm}^{60}$. Although frequently asymptomatic, prognosis is comparable to that of malign mutations of the cardiac $\beta$ myosin heavy chain. Mortality tends to be high among young men. There occurs a high incidence of death, mostly of a sudden character, before the age of $30^{60}$. As a rule, delayed death due to cardiac failure is not observed ${ }^{41}$. At least 6 mutations have a poor prognosis: Ile79Asn, Arg92Gln, Arg 92Trp, Ala104Val, D Glu160 and Int15 $5^{\mathrm{G}-\mathrm{A} 41,56}$. The sole exception is that of Phe110Ile, which affects 13\% of families with a mutation of the cardiac troponin T gene; in Japan it is occasionally associated with apical hypertrophy ${ }^{61}$. As in the benign mutations of the $\operatorname{cardiac} \beta$ myosin heavy chain, no change occurs in the charge of the amino acid involving one of the main sites of binding with the cardiac $\alpha$ actin molecule.

It has been possible by reanalysis of the genome organization of the gene of cardiac troponin $\mathrm{T}$ to identify codon 102 as the point susceptible to mutation ${ }^{57}$. Distinct characteristics were observed in one of the mutations involving this codon, Arg102Leu, in which high penetrance with a marked degree of hypertrophy was noted.

A mutation of the cardiac troponin $T$ gene associated with extensive myofibrillar disarray without an increase in cardiac mass has also been reported in a family with sudden death ${ }^{13}$. The mutation involves exon 9 with a substitution of arginine by leucine in codon 94, producing an exchange of amino acid charge and protein conformation change. It can be deduced that hypertrophy may not be the factor determining sudden death in hypertrophic cardiomyopathy but rather, the myofibril disarrangement associated with fibrosis. In a contemporaneous form, mutation Arg278Cys has been described in a 67-year-old subject who 10 years ago was normal from clinical and electrocardiogram points of view ${ }^{62}$. All known cases until then had begun in adolescence. It is concluded that mutation of cardiac troponin $\mathrm{T}$ can become clinically manifest later in life, justifying cases of sudden death in middle-aged, previously asymptomatic individuals, with a normal or slightly hypertrophic left ventricle.

Mutations of the $\alpha$ tropomyosin gene - The evaluation of families that did not exhibit a linkage with CMH1 and $\mathrm{CMH} 2$ facilitated the identification by Thierfelder et al in 1993 of a third locus, mapped on chromosome 15q22, called CMH3. The gene composed of 14 exons, which encodes a tropomyosin, is localized in the long arm of this chromosome and transcribes $1 \mathrm{~kb} \mathrm{RNAm}^{28}$. It is only implicated in the development of a few, about 5\%, of the cases of the disease ${ }^{41}$.

$\alpha$ tropomyosin is a 284 amino acid polypeptide, which constitutes the thin filaments and is abundantly expressed in the skeletal musculature. It represents $5 \%$ of the myofibril protein. Localized in a major groove of the actin filament, it has a preponderant role in the composition and organization of the thin filaments by establishing the binding between the troponin complex and actin ${ }^{42}$.

Mutations of this gene, numbering 4 , are all missense mutations. Initially 2 mutations on exon 5 were described, Asp175Assn and Glu180Gly ${ }^{41,56}$. Later, Ala63Val and 
Lys70Trh were reported ${ }^{63}$. Some of these mutations can interfere with calcium regulation in the thin filaments. Contrary to mutations of other genes, they are expressed in various structures like gametes, lymphocytes, and myocytes, but being only capable of producing myocardial disease ${ }^{64}$.

Mutation Asp175Asn seems to predominate and produced a mutated protein expressed in the myocardium and skeletal musculature ${ }^{65}$. The recurrent identification of the G-A transition in exon 5 indicates a high propensity toward mutagenesis in the 579 residue of $\alpha$ tropomyosin ${ }^{65}$.

De novo mutation involving the $\alpha$ tropomyosin gene have been reported. They can result in transmission to descendants and can cause familial hypertrophic cardiomyopathy, as already referred to in relation to mutation Asp175Asn ${ }^{64}$. Carriers of apparently sporadic forms of mutation of this gene should be warned about the risk of transmission. Spontaneous mutations appear precociously in gamete development or during embryonic life.

The clinical consequences of mutations of the $\alpha$ tropomyosin gene are less known. The phenotype is characterized by generally mild hypertrophy ${ }^{56}$, but variations in the degree and extension of the hypertrophy between carriers of the same mutation have been observed ${ }^{65,66}$. From the histopathological point of view, no large difference relative to gene mutation encoding proteins composing the thick filaments exists ${ }^{65}$. In Asp175Asn, hypertrophy is of a variable degree and extension among members of distinct families ${ }^{65}$. Prognosis is favorable, with a life expectancy close to normal, comparable to that with benign mutations of the $\beta$ myosin heavy chain. Glu180Gly produces less hypertrophy, but has a similar survival curve ${ }^{56}$. Ala63 Val and Lys70Thr are described as having a less favorable prognosis, with evolution towards cardiac failure ${ }^{66}$. Therefore, in relation to the $\alpha$ tropomyosin gene, the type of mutation determines the clinical expression.

Mutations of the gene of the myosin-binding protein C CMH4 - In 1993, a new locus situated on the 11p11.2 chromosome called CMH4, was identified ${ }^{67}$. Subsequently, Watkins et al ${ }^{34}$ simultaneously with Bonne et al ${ }^{35}$ described the first 3 mutations of the gene encoding the myosinbinding $\mathrm{C}$-protein in unrelated families, considered as causing the disease.

The myosin-binding protein $\mathrm{C}$ is a myofibril protein that does not directly participate in contraction. Its expression is restricted to the myocardium. It is placed transversally in the A bands and binds the myosin of the thick filaments and titin of the elastic filaments. Its function is little known, but it modulates speed of con-traction in response to adrenergic stimulation ${ }^{34,42}$.

The sequence and organization of the gene have been recently described. Formed by the $21 \mathrm{~KB}$ DNA, it is made up of 35 exons ${ }^{42}$. Analysis of the genetic linkage shows that $20 \%$ of the cases of hypertrophic cardiomyopa- thy are caused by this gene ${ }^{68}$. About 28 distinct mutations have been described in unrelated families. Missense mutations and nonsense mutations, in which a single substitution of a base of the DNA leads to the appearance of the terminal codon, precociously closing the synthesis of protein, have been reported ${ }^{34,35,69}$. Mutations by deletion, insertion, duplication, or splicing have also been documented ${ }^{34,35,69,70}$. The consequences of the mutations on the structure and function of the protein are as yet not well known.

Myosin-binding protein $\mathrm{C}$ is composed of 1,274 amino acids, required for its incorporation into the sarcomere ${ }^{69}$. Mutations with amino acid exchange presumably act by a dominant negative effect. Others like duplicating or splice site mutations modify the reading matrix of the DNA, generating truncated proteins not having the terminus responsible for the binding to myosin ${ }^{34,69,70}$. The truncated protein can bind in a defective manner to myosin or not be incorporated into A band of the sarcomere, preventing the interaction of the contractile unit with the cytoskeleton ${ }^{69}$. Another possibility is that due to the low stability of the transcript, because no production occurs of the stable protein, the mutation acts as an null allele ${ }^{69}$. In the recently described splice site mutation, the isolated insertion of guanine in exon 25 leads to the loss of 40 bases on the mRNA, which is then processed in an abnormal manner ${ }^{70}$. The truncated protein generated could not be isolated by endomyocardial biopsy, presumably because it is expressed at very low concentrations or by not being present. Insufficient synthesis or rapid degradation may justify lack of detection of the mutated protein.

Mutations of the gene of the myosin-binding protein $\mathrm{C}$ have incomplete penetrance with mild hypertrophy and a generally favorable clinical evolution. Many carriers of the genotype do not exteriorize the phenotype, revealing normal thickness on echocardiography and, frequently, absence of electrocardiographic alterations ${ }^{69}$. Only $58 \%$ of the affected members express some degree of hypertrophy prior to reaching 50 years of age ${ }^{69}$. A tendency exists for penetration to remain incomplete until the $5^{\text {th }}$ or $6^{\text {th }}$ decades of life. Phenotype expression although delayed is usually uniform.

Mutations of this gene go against the conception that carriers of the disease should develop clinical manifestations of it prior to the beginning of adulthood. Late manifestation may render diagnosis of familial forms and identification of a autosumal dominant inheritance difficult. Despite the tendency towards benign evolution with a more favorable survival curve in comparison to mutations of other genes, increased mortality occurs conditioned by age and the development of hypertrophy ${ }^{69}$. The greatest portion of deaths related to cardiopathy have a sudden character. From the moment when it becomes clinically manifest, it does not have a truly benign character. In a recent evaluation ${ }^{68}$, prognosis was more favorable than in mutations of 
the cardiac $\beta$ myosin heavy chain, no deaths prior to 40 years of age having been recorded. In the SASint 20 mutation, cumulative survival of $90 \%$ at 50 years was situated in the intermediary band relative to mutations Arg403Leu and Arg403Thr of the cardiac $\beta$ myosin heavy chain, which reached $42 \%$ and $100 \%$ survival, respectively ${ }^{71}$.

The lower clinical repercussion of mutations of myosin-binding protein $\mathrm{C}$ is attributable to the predominance of the synthesis of the truncated protein not assimilated by the sarcomere ${ }^{71}$. Nevertheless, due to the possible occurrence of exceptions, as recorded in mutations of other genes, it is necessary to confirm the phenotype by the evaluation of larger families. Due to the late beginning, the benign evolution and the predominance of asymptomatic individuals with mild hypertrophy, the prevalence of these mutations may be underestimated. Therefore, screening of patients suspected of having hypertrophic cardiomyopathy should include individuals of all age groups, as well as adults considered to be healthy in previous clinical evaluations ${ }^{72}$.

Mutations of the genes of the essential and regulatory myosin light chains - Mutations of the genes that encode, respectively, the essential and regulatory myosin light chains were recognized in 1996 as being associated with hypertrophic cardiomyopathy ${ }^{36}$. Myosin is a hexameric protein, formed by 2 heavy chains and 2 pairs of light chains, designated as essential and regulatory. The essential myosin light chain is a polypeptide with 195 amino acids expressed in the myocardium and skeletal muscle ${ }^{42}$. It has inotropic properties, probably related to the activation of the thin filaments. When removed from skeletal muscle in vitro, it reduces the speed of movement of actin and the generation of isometric force ${ }^{42}$. The regulatory myosis light chain, composed of 166 amino acids, is expressed in the same structures ${ }^{42}$. Rupture of the gene results in sarcomere disorganization and gives rise to the embryonic form of dilated cardiomyopathy ${ }^{73}$. The light chains could have the function of stabilizing the structure that bears the globular head of the protein in cardiac $\beta$ myosin heavy chain molecule ${ }^{42}$.

Considering that mutations of the cardiac $\beta$ myosin heavy chain affect the interface with the light chains, Poetter et al ${ }^{36}$ proposed the hypothesis that this protein when mutated-could cause the disease. To this effect, the DNA of 383 unrelated families affected by hypertrophic cardiomyopathy was screened. The amplification of 7 exons that make up the gene of the light chain of essential myosin located in chromosome $3 p$ revealed 2 point mutations with amino acid substitution. Six of 13 affected members with mutation Met149Val had the same phenotype, characterized by hypertrophy of papillary muscles and adjacent myocardium, generating medial ventricular obstruction. This less frequent form had not been previously demonstrated to have familial character. This mutation demonstrated high penetrance and a poor prognosis. Screening of another 16 unrelated individuals identified mutation Arg154His associated with the same phenotype ${ }^{36}$.

Distinct mutations involving the gene of the regulatory myosin light chain have been simultaneously described by the same research group ${ }^{36}$. The gene mapped in chromosome $12 \mathrm{q}$ is distributed in $12 \mathrm{~KB}$ of DNA and covers 7 exons ${ }^{42}$. Three mutations with amino acid exchange have been documented: Ala13Thr, Glu22 Lys, and Pro94Arg, equally associated with mid-ventricular obstructive forms. The expression of these mutations was shown to be variable and of decreasing penetrance. Two other mutations associated with typical familial forms have subsequently been described ${ }^{74}$.

Mutations of the light chains of respectively essential and regulatory myosin, determine variable phenotypes between families and between members of the same family. Mutations of both genes are associated with myopathic alterations, documented on skeletal muscle biopsy ${ }^{36}$.

Mutations of the cardiac troponin I gene - In 1997, Kimura et al described the $7^{\text {th }}$ gene responsible for hypertrophic cardiomyopathy, encoding cardiac troponin I ${ }^{37}$. Localized on chromosome 19p, the gene is constituted of 6.2 KB DNA arranged in 8 exons. Cardiac troponin I is a polypeptide formed by 210 amino acids, integrating the troponin complex of the thin filaments ${ }^{42}$. It exerts a modulator influence on the calcium-dependent actin-myosin interaction. The region of the molecule containing the site binding to actin is essential for the protein to exert inhibitory action on contraction. The $\mathrm{C}$ terminal, in turn, effects the binding to troponin $\mathrm{C}^{42}$.

Six mutations have been originally described in this gene, 5 missense mutations and one deletion without disruption of the reading frane ${ }^{37}$. Recently, deletion on exon 8 producing loss of 8 amino acids and of the stop codon was described for the first time in a Western family ${ }^{75}$. Because the protein has its expression restricted to the myocardium, the effect of the mutations is limited to the heart. The mechanisms of action of these mutations are still hypothetical. They reach exons 7 and 8 , corresponding to terminal $C$ of the molecule ${ }^{42}$. In this way, they become capable of interfering with the inhibitory function exerted by the protein and condition the loss of regulation of the contractile function, leading to hypercontractibility ${ }^{37}$. Therefore cardiac troponin I mutations may act by mechanisms distinct from those of the other mutations.

Mutations of troponin I genes have a heterogeneous character. Although some are associated with typical forms of hypertrophic cardiomyopathy, others like Arg 162Trp and Gly203Ser determine apical hypertrophy ${ }^{37}$. Occasional association with Wolff-Parkinson-White syndrome has been noted ${ }^{37}$. It is possible to individualize cases of typical forms and also of apical hypertrophy in the same family among carriers of the same mutation. Apical hypertrophic cardiomyopathy, predominating in the Orient, was preliminarily considered as sporadic in nature. Its identification 
among carriers of mutations of cardiac troponin I and myosin binding protein $\mathrm{C}$ indicated that the apical form is part of the wide clinical spectrum of hypertrophic cardiomyopathy as a sarcomere disease.

A report exists of spontaneous mutation with substitution of lysine for glutamine in codon $206^{37}$. In the typical forms described in the West, hypertrophy has a mild or moderate degree with septal thickness of 15 or $16 \mathrm{~mm}$, or evidence of a subclinical character with expression restricted to the electrocardiogram ${ }^{75}$.

Mutation of the cardiac $\alpha$ actin gene-The gene encoding $\alpha$ cardiac actin was identified in 1999 by Mogensen et $\mathrm{al}^{38}$ as related to the disease in a family with hypertrophic cardiomyopathy. The major protein of the thin filaments, actin is directly involved in the generation of the contractile force of the sarcomere as well as in its transmission to the cell medium. The point mutation of the gene, mapped on chromosome 15, consists of the substitution of guanine for thymine in exon 5 with exchange of alanine by serine in codon 295. The mutation is located on the surface of the protein molecule close to the site of binding to $\beta$ myosin. It is possible that it causes distortion of the topology of the polypeptide, harming the interaction with the thick filaments and the generation of contractile force in the sarcomere. The mutation related to hypertrophic cardiomyopathy is located close to 2 other point mutations recently associated with familial forms of dilated cardiomyopathy. The cardial active gene is the first one to be simultaneously related to 2 distinct forms of cardiomyopathy ${ }^{38}$.

Cardiomyopathy produced by this gene has high penetrance with a predominance of asymptomatic forms and beginning in variable age groups. The phenotype is heterogeneous. Records exist of massive septal hypertrophy, late evolution towards serious systolic dysfunction, and an association with the Wolff-Parkinson-White syndrome in the presence of borderline left ventricular hypertrophy. Many carriers of the genotype exhibit only prominent electrocardiographic alterations at the basal parts of the septum ${ }^{38}$.

The development of myocardial hypertrophy - The mechanisms by which sarcomere protein mutations determine myocardial hypertrophy are still of a speculative character. The major part of information about the physiopathology of mutations is based on transgenic experimental models and in vitro analyses. At the molecular level, mutations determine structural and functional alterations in the sarcomere, which impair the generation of contractile force 28,42,56,59. $^{\text {. }}$ Reduction in contractility, however, does not have a reflex on the global performance of the left ventricle, which characteristically is hyperdynamic or normal. Myocardial hypertrophy, of adaptive and compensatory character, would develop by a series of as yet unknown processes, presumably common to all mutations ${ }^{42,76}$.

It is acknowledged that hypertrophy distribution depends more on hemodynamic factors than on the regio- nal expression of mutated protein ${ }^{28}$. Considering the biventricular expression of mutated protein, it is not clear why, in the majority of cases, hypertrophy is restricted to the left ventricle and predominates at the level of the interventricular septum. The higher-pressure regimen and the action of modifying genes ${ }^{28,42,66}$ possibly establish left ventricular hypertrophy. The DD genotype of the angiotensin-converting enzyme is found in association with the higher incidence of sudden death and extensive, diffuse hypertrophy ${ }^{77}$. Angiotensin II by its mitogenic action influences the development of hypertrophy. The reduction in contractility conditioned by the insertion of mutated proteins into the sarcomere increases stress at the level of the myocardial fiber, resulting in the expression of genetic factors that induce cell hypertrophy ${ }^{76}$. It is possible that the DD genotype directly influences the phenotypic expression and the degree of penetrance ${ }^{28}$. However, some manifestations, such as elongation and the anomalous insertion of mitral valve leaflets, the thickening of the intramural arteries, and the deposition of collagen in the extracellular matrix, seem not to be directly related to these mechanisms. The possibility that trophic and mitogenic factors may influence these processes cannot be discarded ${ }^{76}$.

Essentially, disturbances evidenced at the molecular and cellular levels depend on the gene affected and the type of mutation occurring. Genes causing the disease encode sarcomere proteins directly involved in the contractile process, as well as polypeptides exerting only a regulatory effect on inotropism. Missense mutations generate anomalous proteins, which supposedly interfere with sarcomere function by a dominant negative effect ${ }^{14,45}$. In cases of deletion or splice site mutation involving changes in the reading frame, truncated proteins that would not be assimilated by the sarcomere are produced ${ }^{70}$. Therefore, protein synthesis would be effected by the normal allele, possibly resulting in a quantitative deficiency of the gene product ${ }^{42}$. The phenotype variation of the same mutation would presumably be due to the intervention of modifying genes, or as yet little known polymorphism, or to the influence of environmental factors like exercise and physical conditioning ${ }^{42}$.

Genetic diagnosis - Genotyping becomes the rational basis of genetic counseling and the identification of patients with a high risk of sudden death, requiring precocious therapeutic intervention. Genetic diagnosis among children and adolescents is decisive in cases of reduced or absent penetrance, or ambiguous situations like those of athletes or persons with systemic arterial hypertension and left ventricular hypertrophy. In affected families, it enables the release of normal members and the precocious identification of malignant mutations. However, genetic analysis is restricted to a few research centers having as their principal objective the identification of new genes and mutations. Large-scale application of information arising from genetic diagnosis in clinical and ethical contexts is still a complex process in view of the risk of doubtful interpretations and unjustified discrimination. 


\begin{tabular}{|ll|}
\hline \begin{tabular}{c} 
Chart I $-\begin{array}{c}\text { Clinical characteristics of hypertrophic cardiomyopathy } \\
\text { in its respective genetic forms }\end{array}$ \\
\hline Gene
\end{tabular} & Characteristics \\
\hline$\beta$ cardiac myosin heavy chain & $\begin{array}{l}\text { Predominance of high penetrance } \\
\text { and severe hypertrophy. } \\
\text { Variable risk of sudden death } \\
\text { according to the mutation. }\end{array}$ \\
Cardiac T troponin & $\begin{array}{l}\text { Low penetrance, mild hypertrophy } \\
\text { associated to early sudden death. }\end{array}$ \\
$\alpha$ tropomyosin & $\begin{array}{l}\text { Large phenotype variation, } \\
\text { predominance of mild hypertrophy } \\
\text { variable prognosis. }\end{array}$ \\
Myosin-binding protein C & $\begin{array}{l}\text { Incomplete and late penetrance, } \\
\text { mild hypertrophy and benign evolution. }\end{array}$ \\
Essential and regulatory & $\begin{array}{l}\text { High penetrance, mid } \\
\text { ventricle obstruction and } \\
\text { myosin light chain }\end{array}$ \\
typical forms, bad prognosis \\
Cardiac I troponin & $\begin{array}{l}\text { Heterogeneous phenotype, apical } \\
\text { hypertrophy and typical forms }\end{array}$ \\
$\alpha$ cardiac actin & $\begin{array}{l}\text { High penetrance, beginning at } \\
\text { various ages, heterogeneous phenotype }\end{array}$ \\
\hline
\end{tabular}

Genetic studies have made a decisive contribution by enabling the characterization of hypertrophic cardiomyopathy as a sarcomere disease and identification of the predominance of familial forms. The spectral character of the disease becomes evident from the corroboration that in affected families $20 \%$ to $30 \%$ of the carriers of the genotype do not meet classical echocardiographic criteria of left ventricular hypertrophy, even though being susceptible to sudden death ${ }^{10,11,14,70}$. In this group, the record of minimal alterations on the electrocardiogram and echocardiogram have greater significance than in the population in general ${ }^{10,78}$. One verifies that prognosis is determined by the genetic substrate and that phenotype and clinical evolution vary according to the gene and type of mutation (Table I).

The expectation for the coming years is that remaining genes and their respective mutations will be identified. The evaluation of the effect of different mutations on the structure and function of the sarcomere is equally decisive, depending above all on the expansion of experimental studies based on transgenic animals. It is of fundamental importance to expand the analysis of genotype-phenotype relationships, still limited by the small number of families evaluated and by allele heterogeneity. The identification of modifying genes and of the mechanisms implicated in the establishment of myocardial hypertrophy should be effected, probably over the long range. Elucidation of these processes is essential for the introduction in the future of genetic therapy as a decisive form of intervention in the development of hypertrophic cardiomyopathy.

\section{References}

1. Richardson P, McKenna WJ, Bristow M, et al. Report of 1995 World Health Organization/International Society and Federation of Cardiology task force on the definition and classification of cardiomyopathies. Circulation 1996; 93: 841-2.

2. Maron BJ, Garder JM, Flack JM, et al. Prevalence of hypertrophic cardiomyopathy in a general population of young adults: echocardiographic analysis of 4111 subjects in the CARDIA study. Circulation 1995; 92: 785-9.

3. Frank S, Braunwald E. Idiopathic hypertrophic subaortic stenosis: a critical analysis of 126 patientes with emphasis on the natural history. Circulation 1968; 37: 759-88.

4. Brock R. Functional obstruction of left ventricle (acquired subvalvar aortic stenosis). Guy's Hosp Rep 1957; 106: 221-38

5. Teare D. Asymmetrical hypertrophy of the heart in young adults. Br Heart J 1958; 20: $1-8$.

6. Goodwin JF. Hypertrophic cardiomyopathy: a disease in search of its own identity. Am J Cardiol 1980; 45: 177-80.

7. Maron BJ, Gottdiener JS, Epstein SE. Patterns and significance of left ventricular hypertrophy in hypertrophic cardiomyopathy: a wide range two-dimensional echocardiographic study of 125 patients. Am J Cardiol 1981; 48: 418-28.

8. Wigle D, Sasson Z, Handerson MA, et al. Hypertrophic cardiomyopathy: the importance of the site and the extent of hypertrophy. Prog Cardiovasc Dis 1985; 28: 1-83.

9. Klues H, Schiffers A, Maron BJ. Phenotypic spectrum and patterns of left ventricular hypertrophy in hypertrophic cardiomyopathy: morphologic observations and significance as assessed by 2-dimensional echocardiography in 600 patients. J Am Coll Cardiol 1995; 26: 1699-708.

10. Hagège AA, Dubourg O, Desnos M, et al. Familial hypertrophic cardiomyopathy. Cardiac ultrasonic abnormalities in genetically affected subjects without echocardiographic evidence of left ventricular hypertrophy. Eur Heart J 1998; 19: 490-9.

11. Maron BJ. Hypertrophic cardiomyopathy. Lancet 1997; 350: 127-33.

12. Sharma S, Elliott PM, Whyte G, Mahon N, Virdee M, McKenna WJ. Utility of metabolic exercise testing in distinguishing hypertrophic cardiomyopathy from physiologic left ventricular hypertrophy in athletes. J Am Coll Cardiol 2000; 36: 864-70.

13. Varnava A, Baboonian C, Davison F, et al. A new mutation of cardiac troponin T causing familial hypertrophic cardiomyopathy without left ventricular hypertrophy. Heart 1999; 82: 621-4.

14. McKenna WJ. The future in hypertrophic cardiomyopathy: important clues and potential advances from understanding of the genotype phenotype relationship. Ital Heart J 2000; 1: 17-20.

15. Schwartz K, Carrier L, Guicheney P, Komadja M. Molecular basis of familial hypertrophic cardiomyopathy. Circulation 1995; 91: 532-40.

16. Maron BJ, Moller JH, Seidman SE, et al. Impact of laboratory molecular diagnosis on contemporary diagnostic criteria for genetically transmitted cardiovascular diseases: hypertrophic cardiomyopathy, long-QT syndrome, and Marfan syndrome. Circulation 1998; 98: 1460-71.

17. Maron BJ, Tajik AJ, Ruttemberg HJ, et al. Hypertrophic cardiomyopathy in infants: clinical features and natural history. Circulation 1982; 65: 7-17.

18. McKenna WJ, Deanfield J, Faruqui A, England D, Oakley C, Goodwin J. Prognosis in hypertrophic cardiomyopathy: role of age, clinical, electrocardiographic and hemodynamic features. Am J Cardiol 1981; 47: 532-8.

19. Spirito P, Maron BJ, Bonow RO, Epstein SE. Ocurrence and significance of progression of left ventricular wall thinning and relative cavity dilatation in hypertrophic cardiomyopathy. Am J Cardiol 1987; 60: 123-9.

20. Lewis JF, Maron BJ. Clinical and morphological expression of hypertrophic cardiomyopathy in patients with > 65 years of age. Am J Cardiol 1994; 73: 1105-11.

21. Maron BJ, Bonow RO, Cannon RO, Leon MB, Epstein SE. Hypertrophic cardiomyopathy: interrelation of clinical manifestations, pathophysiology and therapy. N Engl J Med 1987; 316: 780-9, 844-52.

22. Spirito P, Chiarella F, Carratino L, Zoni-Berisso M, Belloti P, Vecchio C. Clinical course and prognosis of hypertrophic cardiomyopathy in an outpatient population. N Engl J Med 1989; 320: 749-55.

23. Artega E, Ianni BM, Fernandes F, Wajsbrot DB, Mady C. Long-term follow-up of patients with hypertrophic cardiomyopathy. Circulation 1999; 100: I-77.

24. Maron BJ, Casey SA, Poliac L, Gohman TE, Almquist AK, Aeppli DM. Clinical course of hypertrophic cardiomyopathy in a regional US cohort. JAMA 1999; 281: 650-5.

25. Spirito P, Seidman SE, McKenna WJ, Maron BJ. The management of hypertrophic cardiomyopathy. N Engl J Med 1997; 336: 775-85. 
26. Watkins H. Sudden death in hypertrophic cardiomyopathy. N Engl J Med 2000; 342: 422-4.

27. Maron BJ, Nichols PF, Pickle LW, Wesley YE, Mulvihill JJ. Patterns of inheritance of hypertrophic cardiomyopathy: assessment by M-mode and two-dimensional echocardiography. Am J Cardiol 1984; 53: 1087-94.

28. Marian AJ, Roberts R. Recent advances in molecular genetics of hypertrophic cardiomyopathy. Circulation 1995; 92: 1336-47.

29. Fananapazir L, Epstein ND. Prevalence of hypertrophic cardiomyopathy: limitations of screening methods. Circulation 1995; 92: 700-4.

30. Jarcho JA, McKenna WJ, Pare JAP, et al. Mapping a gene for familial hypertrophic cardiomyopathy to chromosome 14q1. N Engl J Med 1989; 321: 372-8.

31. Geisterfer-Lowrance AA, Kass S, Tanigawa G, et al. A molecular basis for familial hypertrophic cardiomyopathy: a beta-myosin heavy chain gene missense mutation. Cell 1990; 62: 999-1006.

32. Watkins H, MacRae C, Thierfelder L, et al. A disease locus for familial hypertrophic cardiomyopathy maps to chromosome 1q3. Nat Genet 1993; 3: 333-7.

33. Thierfelder L, MacRae C, Watkins $\mathrm{H}$, et al. A familial hypertrophic cardiomyopathy locus maps to chromosome 15q2. Proc Natl Acad Sci USA 1993; 90: 6270-4.

34. Watkins H, Conner D, Thierfelder L, et al. Mutations in the cardiac myosin binding protein-C on chromosome 11 cause familial hypertrophic cardiomyopathy. Nat Genet 1995; 11: 433-7.

35. Bonne G, Carrier L, Bercovici J, et al. Cardiac myosin binding protein-C gene splice acceptor site mutation is associated with familial hypertrophic cardiomyopathy. Nat Genet 1995; 11: 438-40.

36. Poetter K, Jiang H, Hassanzadeh S, et al. Mutations in either the essential and regulatory light chains of myosin are associated with a rare myopathy in human heart and skeletal muscle. Nat Genet 1996; 13: 63-9.

37. Kimura A, Harada H, Park JE, et al. Mutations in the cardiac troponin I gene associated with hypertrophic cardiomyopathy. Nat Genet 1997; 16: 379-82.

38. Mogensen J, Klausen IC, Pedersen AK, et al. $\alpha$ cardiac actin is a novel disease gene in familial hypertrophic cardiomyopathy. J Clin Invest 1999; 103: R39-R43.

39. MacRae CA, Ghaisas N, Kass S, et al. Familial hypertrophic cardiomyopathy in Wolff-Parkinson-White syndrome maps to a locus in chromosome 7q3. J Clin Invest 1995; 96: 1.216-1.20.

40. Hoffee P. Genética Médica Molecular. Rio de Janeiro: Guanabara. Koogan, 2000

41. Watkins H, McKenna WJ, Thierfelder L, et al. Mutations in the genes for cardiac troponin T and a tropomyosin in hypertrophic cardiomyopathy. N Engl J Med 1995; 332: 1058-64.

42. Bonne G, Carrier L, Richard P, Hainque B, Schwartz K. Familial hypertrophic cardiomyopathy: from mutations to functional defects. Circ Res 1998; 83: 580-93.

43. Marian AJ, Yu QT, Mares A, Hill R, Roberts R, Perryman MB. Detection of a new mutation in the $\beta$-myosin heavy chain gene in an individual with hypertrophic cardiomyopathy. J Clin Invest 1992; 90: 2156-65.

44. Schwartz K. Familial hypertrophic cardiomyopathy: nonsense x missense mutations. Circulation 1995; 91: 2865-7.

45. Watkins H, Rosenzweig A, Hwang DS, et al. Characteristics and prognostics of myosin missense mutations in hypertrophic cardiomyopathy. N Engl J Med 1992; 326: 1108-14

46. Dausse E, Komadja M, Fetler L, et al. Familial hypertrophic cardiomyopathy: microsatellite haplotyping and identification of a hot spot for mutations in the $\beta$ myosin heavy chain gene. J Clin Invest 1993; 92: 2807-13.

47. Fananapazir L, Dalakas MD, Cyran F, Cohn G, Epstein ND. Missense mutation in the $\beta$-myosin heavy chain gene cause central core disease in hypertrophic cardiomyopathy. Proc Natl Acad Sci USA 1993; 90: 3993-7.

48. Epstein ND, Cohn GM, Cyran F, Fananapazir L. Differences in clinical expression of hypertrophic cardiomyopathy associated with two distinct mutations in the $\beta$-myosin heavy chain gene: a 908Leu-Val mutation and a 403Arg-Gln mutation. Circulation 1992; 86: 345-52.

49. Anan $\mathrm{R}$, Greve $\mathrm{G}$, Thierfelder $\mathrm{L}$, et al. Prognostic implications of novel $\beta$ cardiac myosin heavy chain gene mutations that cause familial hypertrophic cardiomyopathy. J Clin Invest 1994; 93: 280-5.

50. Hwang TH, Lie WH, Kimura A, et al. Early expression of a malignant phenotype of familial hypertrophic cardiomyopathy associated with a Gly716Arg myosin heavy chain mutation in a korean family. Am J Cardiol 1998; 82: 1509-13.

51. Nishi H, Kimura A, Matsumyama K, Koga Y, Sasazuki T, Toshima H. Two distinct mutations of cardiac $\beta$ myosin heavy chain gene found in a Japanese patient with hypertrophic cardiomyopathy. Circulation 1993; 88(suppl I): I-343.

52. Fananapazir L, Epstein ND. Genotype-phenotype correlations in hypertrophic cardiomyopathy: insights provided by comparisons of kindreds with distinct and identical $\beta$-myosin heavy chain gene mutations. Circulation 1994; 89: 22-32.

53. Watkins H, Thierfelder L, Hwang D-J, McKenna WJ, Seidman JG, Seidman CE.
Sporadic hypertrophic cardiomyopathy due to de novo myosin mutations. J Clin Invest 1992; 90: 1666-71.

54. Muraishi A, Kai H, Nishi H, Imaizumi T. Malalignment of the sarcomeric filaments in hypertrophic cardiomyopathy with cardiac myosin heavy chain gene mutation. Heart 1999; 82: 625-9.

55. Cuda G, Fananapazir L, Zhu WS, Seller JR, Epstein ND. Skeletal muscle expression and abnormal function of beta-myosin in hypertrophic cardiomyopathy. $\mathrm{J}$ Clin Invest 1993; 91: 2861-5.

56. Thierfelder L, Watkins H, MacRae C, et al. Alpha-tropomyosin and cardiac troponin T mutations cause familial hypertrophic cardiomyopathy. Cell 1994; 77 : 701-12.

57. Forissier JF, Carrier L, Farza H, et al. Codon 102 of the cardiac troponin T gene is a putative hot spot for mutations in familial hypertrophic cardiomyopathy. Circulation 1996; 94: 3069-73.

58. Varnava AM, Davison F, Cruz F, et al. A de novo mutation of the troponin T gene in a patient with hypertrophic cardiomyopathy. J Am Coll Cardiol 2000; 35(suppl A): 201A

59. Redwood C, Lohmann K, Bing W, et al. Investigation of a truncated cardiac troponin $\mathrm{T}$ that causes familial hypertrophic cardiomyopathy. Circ Res 2000; 86: 1146-52.

60. Moolman J, Corfield VA, Posen B, et al. Sudden death due to troponin T mutations. J Am Coll Cardiol 1997; 23: 549-55.

61. Anan R, Shono H, Kisanuki A, Arima S, Nakao S, Tanaka H. Patients with familial hypertrophic cardiomyopathy caused by a Phe110Ile missense mutation in the cardiac troponin $\mathrm{T}$ gene have variable cardiac morphologies and a favorable prognosis. Circulation 1998; 98: 391-7.

62. Elliot PM, D‘Cruz L, McKenna WJ. Late-onset hypertrophic cardiomyopathy caused by a mutation in the cardiac troponin T gene. N Engl J Med 1999; 341: 1855 .

63. Nakajima C, Matsui H, Nagata S, et al. Novel missense mutation in alpha-tropomyosin gene found in japanese patients with hypertrophic cardiomyopathy. J Mol Cell Cardiol 1995; 27: 2053-8.

64. Watkins H, Anan R, Coviello DA, Spirito P, Seidman JG, Seidman C. A de novo mutation in the $\alpha$-tropomyosin that causes hypertrophic cardiomyopathy. Circulation 1995; 91: 2302-5.

65. Coviello DA, Maron BJ, Spirito P, et al. Clinical features of hypertrophic cardiomyopathy caused by mutation of a "hot spot" in the $\alpha$-tropomyosin gene. J Am Coll Cardiol 1997; 29: 635-40.

66. Fananapazir L. Advances in molecular genetics and management of hypertrophic cardiomyopathy. JAMA 1999; 281: 1746-52.

67. Carrier L, Hengstenberg C, Beckmann JS, et al. Mapping of a novel gene for familial hypertrophic cardiomyopathy to chromosome 11. Nat Genet 1993; 4: 311-3.

68. Charron P, Dubourg O, Desnos M, et al. Clinical features and prognostic implications of familial hypertrophic cardiomyopathy related to the cardiac myosin-binding protein C gene. Circulation 1998; 97: 2230-6.

69. Niimura H, Bachinski L, Sangwatanaroj S, et al. Mutations in the gene for cardiac myosin-binding protein $\mathrm{C}$ and late-onset familial hypertrophic cardiomyopathy. N Engl J Med 1998; 338: 1248-57.

70. Moolman JA, Reiths S, Uhl K, et al. A newly created splice donor site in exon 25 of the MyBP-C gene is responsible for inherited hypertrophic cardiomyopathy with incomplete disease penetrance. Circulation 2000; 101: 1396-402.

71. Charron P, Dubourg O, Desnos M, et al. Genotype-phenotype correlation in familial hypertrophic cardiomyopathy. Eur Heart J 1998; 19: 139-45.

72. St. Martin JS, Epstein JA. Hypertrophic cardiomyopathy: beyond the sarcomere. N Engl J Med 1998; 338: 1303-4.

73. Chen J, Kubalak SW, Minamisawa S, et al. Selective requirement of myosyn light chain in embryonic heart function. J Biol Chem 1998; 273: 1252-6.

74. Flavigny J, Richard P, Isnard R, et al. Identifications of 2 novel mutations in the ventricular regulatory myosin light chain gene (MYL2) associated with familial classic forms of hypertrophic cardiomyopathy. J Mol Med 1998; 76: 208-14.

75. Morner S, Richard P, Kazzan E; Hainque B, Schwartz K, Waldenström A. Deletion in the cardiac troponin I gene in a family from northern Sweden with hypertrophic cardiomyopathy. J Mol Cell Cardiol 2000; 32: 521-5.

76. Marian AJ. Pathogenesis of diverse clinical and pathological phenotypes in hypertrophic cardiomyopathy. Lancet 2000; 355: 58-60.

77. Lechin M, Quiñones MA, Omran A, et al. Angiotensin-I converting enzyme genotypes and left ventricular hypertrophy in patients with hypertrophic cardiomyopathy. Circulation 1995; 92: 1808-12.

78. McKenna WJ, Spirito P, Desnos M, Dubourg O, Komadja M. Experience from clinical genetics in hypertrophic cardiomyopathy: proposal for new diagnostic criteria in adult members of affected families. Heart 1997; 77: 130-2. 